\title{
Three-Dimensional Optical Control of Individual Quantum Dots
}

\author{
Liselotte Jauffred, Andrew C. Richardson, and Lene B. Oddershede*
}

The Niels Bohr Institute, University of Copenhagen, Denmark

Received July 4, 2008; Revised Manuscript Received August 15, 2008

\begin{abstract}
We show that individual colloidal CdSe-core quantum dots can be optically trapped and manipulated in three dimensions by an infrared continuous wave laser operated at low laser powers. This makes possible utilizing quantum dots not only for visualization but also for manipulation, an important advantage for single molecule experiments. Moreover, we provide quantitative information about the magnitude of forces applicable to a single quantum dot and of the polarizability of an individual quantum dot.
\end{abstract}

Colloidal quantum dots (QDs) are fluorescent semiconductor nanocrystals. ${ }^{1}$ They are bright and photostable with a broad excitation spectrum and a narrow emission spectrum, normally distributed around a specific wavelength, dependent on the size of the QD. Absorption of any photons with wavelengths above this specific wavelength causes the formation of an electron-hole pair, the recombination of which results in photon emission. The fluorescent blinking of nanocrystal quantum dots is the result of a bistability between an emitting state where the quantum dot is described as $o$ and the nonemitting off state. ${ }^{2}$ The extreme brightness and photostability of QDs make them excellent choices as markers to visualize biological systems. For instance they have been used to mark individual receptors in cell membranes $^{3}$ or to label living embryos at different stages. ${ }^{4}$

It has long been a goal to optically trap or otherwise control quantum dots ${ }^{5}$ to establish a combined visualization and optical manipulation technique. Optical trapping of aggregates of colloidal quantum dots in two dimensions was recently proved possible ${ }^{6}$ using a pulsed YLF laser, and it was claimed that to trap quantum dots with a continuous wave $(\mathrm{CW})$ laser one would need extremely high powers on the order of $20 \mathrm{~W}$. In this Letter, we prove that optical trapping of individual quantum dots using a $\mathrm{CW}$ infrared laser operated at only $0.5 \mathrm{~W}$ is, in fact, possible. By observing the Brownian motion of the trapped quantum dots, we deduced the strength of the optical trap and thus found the magnitude of the optical forces acting on a single quantum dot. We used two independent approaches to render probable that it was indeed a single quantum dot in the trap and not an aggregate.

An inducible dipole in an inhomogeneous field experiences a force in the direction of the field gradient, the gradient

* Corresponding author, oddershede@nbi.dk. force, $\vec{F}_{\text {grad. }}$. A particle with an induced dipole moment will be forced toward the laser focus by this three-dimensional restoring force. Hence, the existence of an induced QD dipole moment is essential for optical trapping. Opposing the gradient force are the scattering force, $\vec{F}_{\text {scat }}$, and the absorption force, $\vec{F}_{\text {abs }}$, which are proportional to the scattering and absorption cross sections, respectively. If infrared laser light, with a wavelength that exceeds the maximum emitted wavelength of the QDs by far, is used for trapping and if the QDs are physically very small in comparison to the focus area of the trapping laser light, then the scattering and absorption forces are considerably smaller than the gradient force. ${ }^{7}$ Using this approach, we provide an estimate of the polarizability of an individual quantum dot.

In the experiments, the quantum dots were diluted into perfusion chambers made by assembling two coverslips with vacuum grease. We used water-soluble cadmium selenide core, zinc sulfide shell QDs with an emission wavelength of $655 \mathrm{~nm}$ (Invitrogen). The QDs were diluted $1 / 10^{5}$ in $1 \mathrm{M}$ sodium borate $(\mathrm{pH} 8.2)$ with $1 \%$ bovine serum albumin (BSA). This solution was filtered to remove aggregates of QDs and BSA and perfused into the chambers. Afterward, the chambers were sealed with vacuum grease.

The optical tweezers setup was based on a Nd: $\mathrm{YVO}_{4}$ laser (5 W Spectra Physics Millennia, $\lambda=1064 \mathrm{~nm}, \mathrm{TEM}_{00}$ ) implemented in an inverted Leica microscope equipped with a Hg lamp for QD excitation. Furthermore, a quadrant photodiode (S5981, Hamamatsu), allowed for precise position detection with a time resolution of milliseconds. ${ }^{8}$ As described earlier, ${ }^{9}$ to have an efficient trap, we find it important to expand the laser beam such that it only slightly overfills the diameter of the objective. The objective used to focus the laser to a diffraction limited spot was an oil immersion objective (HCX, PL, APO, 100 $\times$, NA $=1.4$ oil CS). Every QD was trapped $3 \mu \mathrm{m}$ above the lower coverslip 


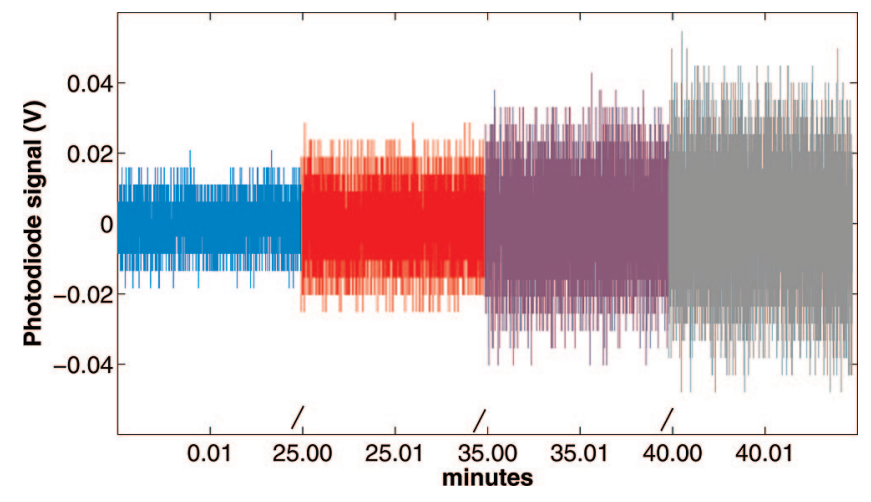

Figure 1. A time series of the photodiode signal. In the beginning (blue) only a single quantum dot is in the trap, and the photodiode signal translates directly to the position of the quantum dot. After about $25 \mathrm{~min}$ (notice the discontinuous time axis), another quantum dot enters the trap and the photodiode signal broadens (red). After 35 min a third particle enters the trap and the signal broadens even further (purple). During the last part of the time series shown (gray) at least four quantum dots are in the trap.

surface. As the experiment takes place in an aqueous environment, spherical aberrations will cause a defocusing of the laser spot in the direction along the propagating laser light. Previously, we devised a method to efficiently cancel spherical aberrations by changing immersion media. ${ }^{10}$ However, the optimal immersion media to cancel spherical aberrations at a depth of $3 \mu \mathrm{m}$ turned out to be fluorescent at the wavelengths used to visualize the QDs. Therefore, we chose to use a conventional immersion media, which still provided a trap strong enough to efficiently trap individual quantum dots. All analyses of our data and the subsequent calculation of the polarizability of a single QD were done in directions perpendicular to the propagating laser light, which are almost not influenced by the presence of spherical aberrations. ${ }^{10}$

The laser power exiting the laser was $0.525 \mathrm{~W}$, and approximately $20 \%$ of this reached the sample. In order to increase the likelihood of trapping only a single QD at a time, we used a very dilute concentration of QDs. Once the experiment was initiated, we typically had to wait for 10 min before a QD would randomly diffuse into the optical trap. The presence of a QD in the trap was clearly visible from reading the voltage signal from the quadrant photodiode collecting the forward-scattered laser light. To record and analyze the emission from the QDs, we used an EMCCD camera (Ixon, Andor) and custom-made MatLab programs.

For experiments where the QDs were specifically attached to a biotinylated coverslip, we used similar QDs as described above but with a streptavidin coating (Invitrogen). The coverslips of the chambers were biotin coated by incubating the samples overnight with BSA-biotin (Sigma) in $20 \mathrm{mM}$ $\mathrm{Na}_{2} \mathrm{HPO}_{4}, 150 \mathrm{mM} \mathrm{NaCl}(\mathrm{pH}$ 7).

The positions visited by individual QDs performing Brownian fluctuations in the optical trap were found as described in refs 8,9 , and 11 . Figure 1 shows the resulting time series and Figure 2 shows the corresponding histograms; the full lines are Gaussian fits to the distributions. After approximately $25 \mathrm{~min}$ the photodiode signal shown in Figure

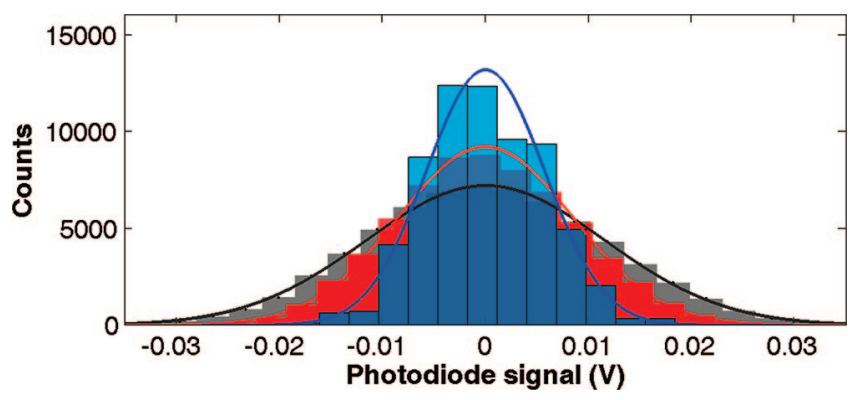

Figure 2. Three histograms from parts of the photodiode signal time series shown in Figure 1. The most narrow histogram (blue) corresponds to the part of the time series where only a single quantum dot is in the trap and where the photodiode signal can be directly translated into the position of the quantum dot. The broader histogram (red) corresponds to the part of the time series with two quantum dots in the trap. The broadest histogram (gray) results from at least four quantum dots in the trap.

1 broadened, after an additional $10 \mathrm{~min}$ it broadened even further, and at later times the broadening continues. Previous experience from trapping gold nanoparticles ${ }^{9}$ suggests that the broadening of the photodiode signal is a result of additional particles entering the trap. Hence, in a typical experiment we would have a single QD at least 10 min before more QDs entered the trap.

As a second and independent way to render probable that we only had a single quantum dot in the trap, we performed an experiment to visualize the blinking of a trapped quantum dot. As earlier described, ${ }^{12}$ this can be investigated by analyzing film sequences of the fluorescence blinking. However, a QD in the optical trap performs considerable Brownian motion within the trap. This complicates visualization of a trapped quantum dot, and it would be difficult to rule out that an observed blinking is not an effect of the QD leaving and entering the focal volume rather than blinking. Therefore, we performed an experiment where a streptavidincoated QD was first trapped and subsequently moved to a biotinylated surface, where it would bind specifically. Figure 3a shows a picture of the coverslip surface before the QD was attached, and Figure $3 b$ shows the same area after the trap with the QD has been lowered and the QD has attached to the surface. Figure $4 \mathrm{a}$ shows the corresponding temporal evolution of the intensity from the attached QD. A clear blinking behavior was observed, thus proving the individuality of the QD previously in the optical trap. Supporting this, Figure $4 \mathrm{~b}$ shows the distribution of the intensities with distinct off and on states.

For positions visited by Brownian fluctuations of an optically trapped particle, it is well established that the optical tweezers exert a harmonic force on the trapped particle: $\vec{F}$ $=-\kappa \vec{x}$, where $\kappa$ denotes the trap stiffness and $\vec{x}$ is the position of the particle with respect to the center of the trap. To quantify $\kappa$ and hence find the range of optical forces exertable on a QD, we performed a power spectral analysis of the time series using the routines described in ref 13 . The distance between the QD and the coverslip surface was very large in comparison to the radius of the QD, and we approximated the overall shape of the QD as a sphere. Hence, the drag coefficient, $\gamma$, was found by Stokes law, $\gamma=6 \pi \eta r, r$ being 

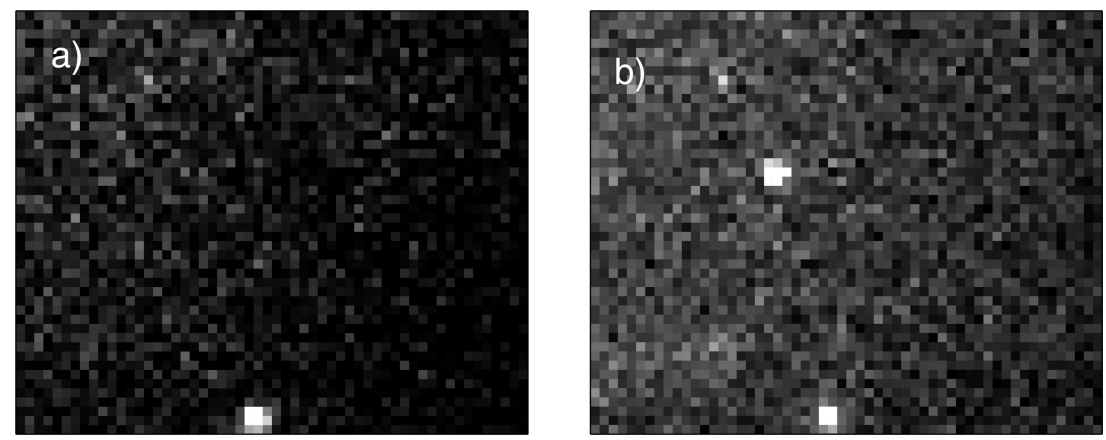

Figure 3. Pictures of a biotinylated surface under the optical trap: (a) the surface before a streptavidin-coated quantum dot is trapped (another quantum dot is attached to the surface); (b) the same part of the surface after lowering a trapped streptavidin-coated quantum dot until it attaches to the surface. The exposure time is $1.8 \mathrm{~s}$, thus integrating over several on states of the quantum dots.
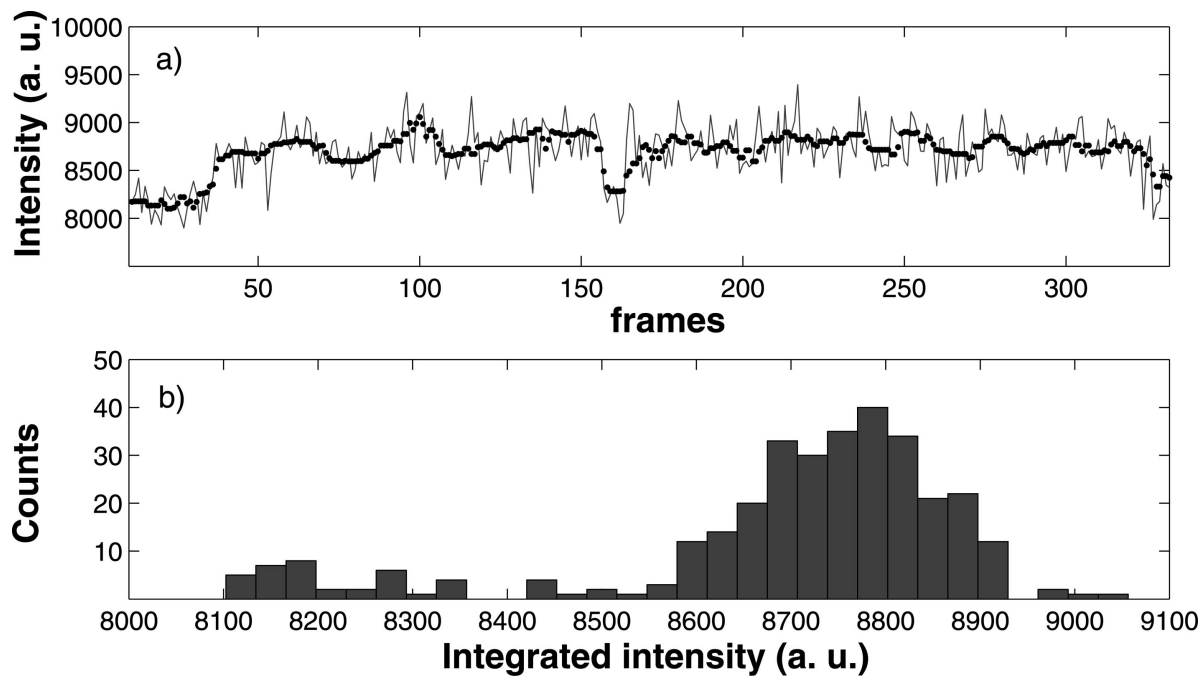

Figure 4. Intensity fluctuations of the attached quantum dot shown in Figure 3b. (a) Time evolution of the intensity. All data points (continuous line) as well as the average from from a sliding window of 10 data points (thick dots) are shown. (b) Corresponding intensity distribution histogram which shows two peaks indicating blinking of the quantum dot.

the effective radius of the QD. The quantum dots had an elliptical core/shell of dimensions $6 \mathrm{~nm}$ (minor axis) and 12 (major axis) ${ }^{14}$ and outside this a shell of stabilizing polymers extending some nanometers out from the particle. In the analysis we assumed an outer effective radius to be used in Stokes law of $15 \mathrm{~nm}$. The equation of motion of a particle performing Brownian fluctuations inside an optical trap is given by the Langevin equation. A Fourier transformation of the Langevin equation gives a positional power spectrum which follows a Lorentzian function. Figure 5 shows the resulting power spectrum of the positions visited by a quantum dot in one lateral dimension; the full line is the best fit by a Lorentzian function taking into account, e.g., filtering effects of the quadrant photodiode ${ }^{15}$ and aliasing effects, ${ }^{13}$ yielding a corner frequency $180 \mathrm{~Hz}$. Analysis of 30 data sets yielded trap stiffnesses in directions perpendicular to the propagating laser beam, $\kappa_{x}$ and $\kappa_{y}$, to be $(2.2 \pm 0.7) \times 10^{-7} \mathrm{~N} / \mathrm{m}$ and $(2.6 \pm 0.6) \times 10^{-7} \mathrm{~N} / \mathrm{m}$, respectively, $y$ being the polarization direction of the laser.

The value of $\kappa$ obtained for a QD can be compared to that obtained from optical trapping of silver and gold nanoparticles of similar sizes..$^{9,11}$ To this end, $\kappa$ should be normalized by the laser power at the specimen, $\sim 0.1 \mathrm{~W}$; hence $\kappa_{\text {norm }}=2.4 \mathrm{pN} / \mathrm{nm} \mathrm{W}$. This value is half the value

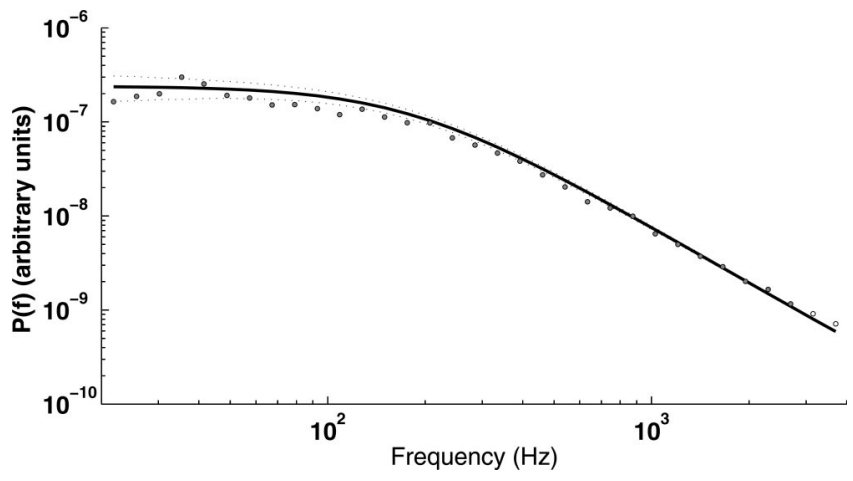

Figure 5. Power spectrum of positions visited by an optically trapped quantum dot in a direction orthogonal to the trapping laser beam. The solid line is the Lorentzian fit to the data and the punctuated lines represent the error bars. The corner frequency of the Lorentzian fit is $180 \mathrm{~Hz}$.

obtained for gold and silver. Therefore, the induced dipole of a QD is somewhat smaller than that of a similarly sized metallic nanoparticle.

As colloidal QDs have only been reported for the last 20 years, many properties are still unknown. Simple questions regarding, e.g., the absolute magnitude of the dipole of a $\mathrm{QD}$, or regarding index of refraction of a QD still remain 
unanswered and perhaps are not even correctly posed. To estimate the polarizability of a single QD, we assumed that the laser beam intensity profile is Gaussian in the two lateral directions and can be described by the following expression where $\sigma$ is the standard deviation of the intensity distribution:

$$
I=I_{0} \mathrm{e}^{-\left(x^{2}+y^{2}\right) 2 \sigma^{2}}
$$

The total power, $\mathbf{P}$, delivered by the laser in the sample equals the integrated intensity

$$
\mathbf{P}=\int_{-\infty}^{\infty} I \mathrm{~d} x \mathrm{~d} y=I_{0} 2 \pi \sigma^{2}
$$

In one dimension the gradient force is ${ }^{7}$

$$
\vec{F}_{\text {grad }}=\frac{\alpha}{2} \nabla\left(\vec{E}^{2}\right)
$$

where $\alpha$ is the polarizability. In fact, it is only the real part of the polarizability which goes into eq 3 , but the imaginary part of $\alpha$, the absorption, is considerably smaller than the real part for the QDs of this study. Therefore, for simplicity, we neglected the contribution from the absorption. $\vec{E}$ is the electric field, and is related to the intensity by

$$
|E|^{2}=2 I / c \varepsilon
$$

where $c$ is the speed of light and $\varepsilon$ the electric permittivity of the medium; we used the wavelength-dependent permittivity of water for a wavelength of $1064 \mathrm{~nm}, \varepsilon=1.32^{2} \varepsilon_{0},{ }^{16}$ $\varepsilon_{0}=8.854 \times 10^{-12} \mathrm{C}^{2} /(\mathrm{J} \mathrm{m})$.

For such a small particle trapped with a wavelength of $1064 \mathrm{~nm}$, far above the emission wavelength of the QD (655 nm), the contributions of $\vec{F}_{\text {scat }}$ and $\vec{F}_{\text {abs }}$ are very small in comparison to the contribution of $\vec{F}_{\text {grad. }}{ }^{7}$ Therefore, we approximate $\vec{F}=-\vec{\kappa} \vec{x}=\vec{F}_{\text {grad }}$.

From our knowledge of $\mathbf{P}$ and $\kappa$, we can use the above equations to find the polarizability, $\alpha$, by combining eqs 1 , 2,3 , and 4 and Taylor expanding for small $x$ while comparing eq 3 to $\vec{F}_{\text {grad }}=-\kappa \vec{x}$. We obtain

$$
\alpha=\frac{2 \pi c \kappa \varepsilon}{P} \sigma^{4}=\left(6.4 \times 10^{-8}\right) \frac{C}{V m^{2}} \sigma^{4}=\left(2.5 \times 10^{-34}\right) \frac{C}{V} m^{2}
$$

Here, $\sigma$ is approximated by the radius of the laser beam at the trap center, ${ }^{7} \sigma \sim 250 \mathrm{~nm}$. Normalized with the permittivity in vacuum, $\varepsilon_{0}$, the polarizability $\alpha / \varepsilon_{0}$ is $2.8 \times$ $10^{7} \AA^{3}$. $\alpha$ is proportional to $\sigma^{4}$; hence, its numerical value is strongly dependent on our somewhat arbitrary choice of the value of $\sigma$. Figure 6a shows the value of $\alpha / \varepsilon_{0}$ for different choices of $\sigma$.

Another way to estimate the polarizability for a dielectric sphere is by the Clausius-Mossotti relation. For this purpose we assumed the CdSe core of the QD to be approximately spherical with a radius, $r_{\text {core }}=5 \mathrm{~nm}$. $\varepsilon=\varepsilon / \varepsilon_{0}$ is the square of the refractive index and $\varepsilon_{\mathrm{qd}}=10$ for CdSe and $\varepsilon=1.74^{16}$ for water at $1064 \mathrm{~nm}$. We find:

$$
\frac{\alpha}{\varepsilon_{0}}=3 V \frac{\varepsilon_{\mathrm{qd}}-\varepsilon}{\varepsilon_{\mathrm{qd}}+2 \varepsilon}=9.6 \times 10^{5} \AA^{3}
$$

This value is significantly smaller than the polarizability deduced above on the basis of the experimentally obtained
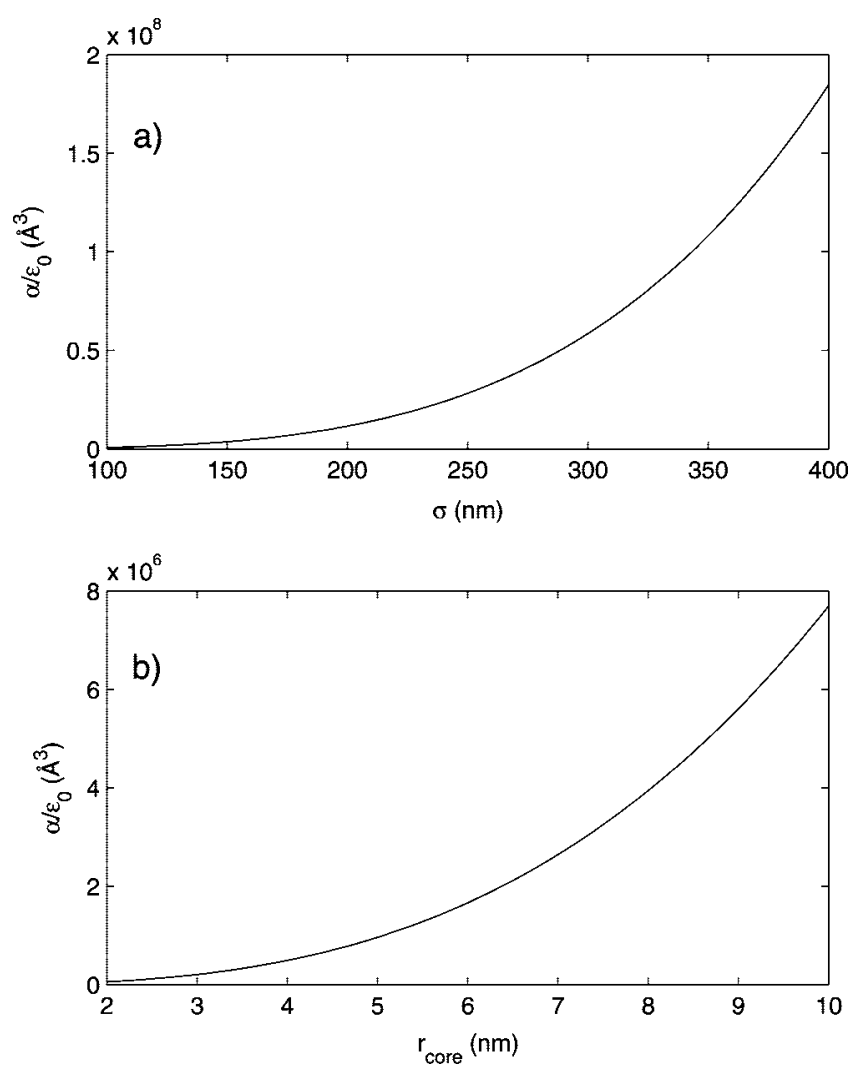

Figure 6. How the value of polarizability, $\alpha / \varepsilon_{0}$ depends on the choices of the trap width $\sigma$ and the QD core radius $r_{\text {core }}$ : (a) in the calculation which is based on the experimentally measured $\kappa$, eq 5; (b) in the calculation based on the Claussius-Mossotti relation, eq 6 .

values of $\kappa$. Hence, a QD probably cannot be approximated as a simple dielectric sphere of $\mathrm{CdSe}$ with the radius of 5 $\mathrm{nm}$. The polarizability given by Clausius-Mossotti would be larger if we took the surrounding shell and polymers into consideration as well. Figure $6 \mathrm{~b}$ shows the dependence of the polarizability on the value of $r_{\text {core. }}$. If one compares parts $\mathrm{a}$ and $\mathrm{b}$ of Figure 6 , it can be seen that by choosing, for instance, $r_{\text {core }}=5 \mathrm{~nm}$ and $\sigma=110 \mathrm{~nm}$, the two different ways of finding the polarizability can be made to coincide. An error in our calculations is that we have chosen to deal with the QD as a sphere even though it is ellipsoidal.

In literature only little quantitative information regarding polarization of individual QDs is available. However, the polarizability has been measured for considerably smaller CdSe QDs (radius $2 \mathrm{~nm}$ ) ${ }^{17}$ to be on the order of $10^{4} \AA^{3}$ and to be strongly dependent on volume; hence, this value could be in accordance with our findings.

Colloidal quantum dots are optimal to visualize biological systems, e.g., as markers for individual proteins ${ }^{1,18}$ or for in vivo studies. ${ }^{4,19}$ Furthermore, QDs themselves possess interesting photonic properties. We have shown threedimensional optical control of individual quantum dots using an infrared CW laser at low laser powers. Two independent methods were employed to show that only a single QD was in the optical trap. Analysis of the Brownian motion of a trapped QD has allowed us to infer the optical forces applicable on a QD to be on the order of tens of femtonewtons for typical excursions. Our findings allow for simul- 
taneous visualization and photonic manipulation of QDs or of molecular systems labeled only with QDs. Additionally, we used the quantitative information regarding the interaction between the electromagnetic field and the QD to calculate an estimate of the absolute polarizability of a colloidal QD; it was found to be $\alpha / \varepsilon_{0}=2.8 \times 10^{7} \AA^{3}$. This value can be refined if one takes into account, e.g., the ellipsoidal shape of the QD, the surrounding shell, and the contribution from absorption. Also, future work will address the issue of how the interaction with the electromagnetic field changes upon changing size of the QD and trapping laser wavelength.

Acknowledgment. We thank Chris Lagerholm, Eva Arnspang Christensen, and S. Nader S. Reihani for important advice, discussions, and suggestions.

\section{References}

(1) Michalet, X.; Pinaud, F.; Lacoste, T. D.; Dahan, M.; Bruchez, M. P.; Alivisatos, A. P.; Weiss, S. Single Mol. 2001, 2, 261-276.

(2) Pelton, M.; Smith, G.; Scherer, N. F.; Marcus, R. A Proc. Natl. Acad. Sci. U.S.A. 2007, 104, 14249-14254.

(3) Dahan, M.; Levi, S.; Luccardini, C.; Rostaing, P.; Riveau, B.; Triller, A. Science 2003, 302, 442-445.

(4) Dubertret, B.; Skourides, P.; Norris, D. J.; Noireaux, V.; Brivanlou, A. H.; Libchaber, A. Science 2002, 298, 1759-1762.
(5) Ramachandran, S.; Merrill, N. E.; Blick, R. H.; van der Weide, D. W. Biosens. Bioelectron. 2005, 20, 2173-2176.

(6) Pan, L.; Ishikawa, A.; Tamai, N. Phys. Rev. B 2007, 75, 161305.

(7) Svoboda, K.; Block, S. M. Opt. Lett. 1994, 19, 930-932.

(8) Oddershede, L.; Grego, S.; Nørrelykke, S. F.; Berg-Sørensen, K. Probe Microsc. 2001, 2, 129-137.

(9) Hansen, P. M.; Bhatia, V. K.; Harrit, N.; Oddershede, L. Nano Lett. 2005, 5, 1937-42.

(10) Reihani, S. N. S.; Oddershede, L. B. Opt. Lett. 2007, 32, 1998-2000.

(11) Bosanac, L.; Aabo, T.; Bendix, P. M.; Oddershede, L. B. Nano Lett. 2008, 8, 1486-1491.

(12) Lagerholm, B. C.; Averett, L.; Weinreb, G. E.; Jacobson, K.; Thompson, N. L Biophys. J. 2006, 91, 3050-3060.

(13) Hansen, P. M.; Tolic-Nørrelykke, I. M.; Flyvbjerg, H.; Berg-Sørensen, K. Comput. Phys. Commun. 2006, 174, 518-520.

(14) Ryman-Rasmussen, J. P.; Riviere, J. E.; Monteiro-Riviere, N. A. J. Invest. Dermatol. 2007, 127, 143-153.

(15) Berg-Sørensen, K.; Oddershede, L.; Florin, E.-L.; Flyvbjerg, H. J. Appl. Phys. 2003, 96, 3167-3176.

(16) Segelstein, D. J. The complex refractive index of water. Master's thesis University of Missouri-Kansas City, Kansas City, MO, 1981.

(17) Wang, F.; Shan, J.; Islam, M. A.; Herman, I. P.; Bonn, M.; Heinz, T. F. Nat. Mater. 2006, 5, 861-864.

(18) Capitanio, M.; Maggi, D.; Vanzi, F.; Pavone, F. S. J. Opt. A: Pure Appl. Opt. 2007, 9, S157-S163.

(19) Larson, D. R.; Zipfel, W. R.; Williams, R. M.; Clark, S. W.; Bruchez, M. P.; Wise, F. W.; Webb, W. W. Science 2003, 300, 1434-1436.

NL801962F 